Analytical Biochemistry: Notes \& Tips

\title{
A general correction to catalytic rates determined for nonprocessive exo-depolymerases acting on both substrate and product in the initial-rate measurement
}

\author{
J. Rose Stoller ${ }^{\mathrm{a}}$, Kurt Wagschal ${ }^{\mathrm{b}}$, Charles C. Lee ${ }^{\mathrm{b}}$, Douglas B. Jordan ${ }^{\mathrm{a}^{*}}$
}

\begin{abstract}
We recently reported on the kinetics of the polygalacturonase $\mathrm{TtGH} 28$ acting on trimer and dimer substrates. When the starting substrate for hydrolysis is the trimer, the product dimer is also subject to hydrolysis, resulting in discrepancies when either the concentration of dimer or monomer product is used for analysis of trimer hydrolysis. Here, we derive a method for determining catalytic rates of exo-hydrolases acting on trimer (and higher order) substrates when products may also be substrates for hydrolysis and show how this correction may be applied for TtGH28.
\end{abstract}

\section{Keywords}


Enzyme kinetics; exo-hydrolase; glycoside hydrolase family 28; nonprocessive; polygalacturonase

\section{Abbreviations}

TtGH28, exo-polygalacturonase from Thermotoga thermophilus; triGalUA, trigalacturonic acid; diGalUA, digalacturonic acid; monoGalUA, galacturonic acid

Enzymatic depolymerization of renewable lignocellulosic and food processing waste biomass to constituent monomeric components for direct use or as platform chemicals is an important bioprocessing approach [1]. Glycoside hydrolases (GH) comprise a large group of enzymes for deconstruction of biomass, currently comprised of 135 families defined by amino acid sequence similarity [2]. For the depolymerization of homogalacturonan derived from pectin to its constituent monomer galacturonic acid, a precursor of galactaric acid [3] as well as ascorbate[4], exo-polygalacturonases have been characterized from both eukaryotes[5-11] and prokaryotes [12-18]. In the course of our studies of the hyperthermostable bacterial polygalacturonase TtGH28 from Thermotoga thermophilus, which is a non-processive exoglycoside hydrolase, we had occasion to determine kinetic constants of the enzyme operating on the trimer substrate trigalacturonic acid (triGalUA) and the dimer substrate digalacturonic acid (diGalUA) [19]. It is desirable to have accurate kinetic constants to help predict enzyme performance under industrial depolymerization conditions. Detection of the enzyme activity was limited to a discontinuous assay method requiring the separation of products from reactant and subsequent detection using high performance anion exchange chromatography with pulsed amperometric detection (HPAEC-PAD) employing a Dionex DX500 HPLC and PA-100 (4x250 
$\mathrm{mm}$ ) anion exchange column coupled with an ED40 PAD, and data analysis was performed using Chromeleon software (Dionex, Sunnyvale, CA). The column was equilibrated with $0.1 \mathrm{~N}$ $\mathrm{NaOH}$, followed by duplicate injections of sample $(25 \mu \mathrm{L})$ and elution using a 5 -min linear gradient $(0.05-0.45 \mathrm{M}$ sodium acetate in $0.1 \mathrm{~N} \mathrm{NaOH})$. The analytes were baseline resolved using this method, and the retention times were $5.5 \mathrm{~min}$ for monoGalUA, $6.4 \mathrm{~min}$ for diGalUA, and 7.2 min for triGalUA. Standard curves were fitted to linear regression using several concentrations between $0.005-0.080 \mathrm{mM}$ of the analytes of interest, with the limit of detection being $0.001 \mathrm{mM}$. In doing so, we immediately noticed a discrepancy between the measured concentrations of the dimer product diGalUA and the monomer product galacturonic acid (monoGalUA) when the enzyme acted on the trimer: unlike the reaction stoichiometry, which indicates equality, the concentration of monoGalUA was greater than that of diGalUA, with more noticeable differences at low concentrations of the starting substrate triGalUA, which indicated strongly the need for a correction to the data to estimate trimer hydrolyzed. Additionally, the initial rate data were fit to the following equation using Grafit [20] to determine kinetic values and standard errors (Fig. 1, wherein duplicate data points obtained for each substrate concentration are shown):

$\frac{v}{E_{t}}=\frac{k_{c a t} S}{K_{m}+S\left(1+\frac{S}{K_{s i}}\right)}$,

where $K_{\mathrm{si}}$ is the substrate inhibition constant. Fig. 1 illustrates the problem that results: for TtGH28 acting on the starting substrate triGalUA, the quantification of monoGalUA produced gives kinetic parameters of $k_{\text {cat }} 9.62 \mathrm{~s}^{-1}$ and $K_{\mathrm{m}} 3.2 \mu \mathrm{M}$ whereas quantification of diGalUA produced gives $k_{\text {cat }} 9.60 \mathrm{~s}^{-1}$ and $K_{\mathrm{m}} 26.7 \mu \mathrm{M}$. The large discrepancy in $K_{\mathrm{m}}$ values determined is purported to result from the action of $\mathrm{TtGH} 28$ on the product diGalUA as well as the starting substrate triGalUA. We derived a method to correct the data to account only for the hydrolysis 
of triGalUA (shown in hollow circles), giving kinetic parameters of $k_{\text {cat }} 9.51 \mathrm{~s}^{-1}$ and $K_{\mathrm{m}} 15.8 \mu \mathrm{M}$. In the following we show how the correction was accomplished. In doing so, we will discuss a more general case for discontinuous assays of an enzyme acting non-processively to hydrolyze a substrate to a product that is also hydrolyzed by the enzyme, as is the case for TtGH28.

When an exo-hydrolase acts on a trimer substrate, hydrolysis results in two products: a monomer and dimer. In a simple case where further hydrolysis of product dimer by the enzyme does not occur to a significant extent, the monomer and dimer concentrations produced by hydrolysis are equivalent to each other and to the concentration of trimer hydrolyzed, allowing for the quantification of trimer hydrolyzed - and thus the catalytic rate of the enzyme acting on the trimer - from the concentration of monomer or dimer produced by discontinuous assay method. However, when hydrolysis of the dimer product also occurs to a significant extent, the concentration of monomer product will be greater than (and the concentration of dimer product will be less than) the concentration of trimer hydrolyzed, resulting in difficulty in accurately determining the turnover of trimer from measuring either monomer or dimer concentrations. Since measuring the disappearance of substrate trimer in a discontinuous assay (a small difference in large numbers) is not as accurate as measuring the appearance of newly formed products, it is advantageous to have a method using the measured concentrations of both products to determine the turnover of trimer hydrolysis without the contribution from dimer hydrolysis. Thus, it is desirable to find a mathematical approach to account for dimer hydrolysis when determining trimer hydrolysis.

In the trimer hydrolysis reaction, when hydrolysis of the product dimer occurs to a large extent, the measured molar concentration of monomer $\left(X_{1}\right)$ is dependent on the molar concentration of trimer hydrolyzed $\left(\triangle X_{3}\right)$, with one monomer released per trimer hydrolyzed, and 
the molar concentration of dimer hydrolyzed $\left(\Delta X_{2}\right)$, with two monomers released per dimer hydrolyzed. Thus, one can state the equation

$X_{1}=\Delta X_{3}+2 \Delta X_{2}$

Additionally, the measured concentration of dimer $\left(X_{2}\right)$ is dependent on the concentration of trimer hydrolyzed $\left(\Delta X_{3}\right)$, with one dimer released per trimer hydrolyzed, and the concentration of dimer hydrolyzed $\left(\Delta X_{2}\right)$, which decreases the concentration of dimer. Thus, one can similarly state another equation,

$X_{2}=\Delta X_{3}-\Delta X_{2}$

which can be rearranged to yield

$\Delta X_{2}=\Delta X_{3}-X_{2}$

Substituting Eq. (4) for $\Delta X_{2}$ into Eq. (2) yields

$X_{1}=\Delta X_{3}+2\left(\Delta X_{3}-X_{2}\right)=3 \Delta X_{3}-2 X_{2}$.

Solving for the concentration of trimer hydrolyzed, $\Delta X_{3}$, gives the following result:

$\Delta X_{3}=2 X_{2} / 3+X_{1} / 3$

Thus, the concentration of trimer hydrolyzed can be quantified from the measured concentrations of the dimer and monomer products using Eq. (6).

This method for quantification of the hydrolysis of a starting substrate by an enzyme when the products of the hydrolysis are also substrates for the enzyme can be expanded for starting substrates of any length. For example, it is easily derived for a tetramer starting substrate that the concentration of tetramer hydrolyzed $\left(\Delta X_{4}\right)$ is

$\Delta X_{4}=3 X_{3} / 4+X_{2} / 2+X_{1} / 4$

Similarly, the concentration of starting substrate pentamer hydrolyzed is given by

$\Delta X_{5}=4 X_{4} / 5+3 X_{3} / 5+2 X_{2} / 5+X_{1} / 5$. 
A more general equation for the concentration of the starting substrate hydrolyzed $\left(\Delta X_{n}\right)$ for a starting substrate of any length $n$ with product molecules of length $i$ can be written as the summation

$\Delta X_{n}=\sum_{i=1}^{n-1} \frac{i X_{i}}{n}$,

where $X_{i}$ is the measured concentration of product of length $i$. The length of starting substrate analyzed using Eq. (9) is mathematically unlimited; however, the practical consideration of the need for separation, detection, and quantification of all products does apply.

The utility of Eq. (6) can be seen in the scenario, described previously, in which the polygalacturonase $\mathrm{TtGH} 28$ acts on the substrate triGalUA as well as the product diGalUA. To determine the rate of hydrolysis of triGalUA, the concentration of triGalUA hydrolyzed in the course of the reaction must be quantified. Three methods of quantification of the concentration of triGalUA hydrolyzed with time were applied to the data using the only concentration of product monoGalUA, only the concentration of product diGalUA, or Eq. (6), which uses both product concentrations. The three methods for quantifying triGalUA hydrolyzed (and thus the rate of catalysis of TtGH28 acting on triGalUA) yields three different steady-state saturation plots (overlaid in Fig. 1), with different apparent values of $K_{\mathrm{m}}$ for TtGH28 acting on triGalUA. Quantification of triGalUA hydrolysis using only the monoGalUA concentration data yields deceptively high rates, particularly at low concentrations of triGalUA, where hydrolysis of product diGalUA becomes more significant and monoGalUA concentrations are higher than resulting from triGalUA hydrolysis alone. Conversely, quantification of triGalUA hydrolysis using only the diGalUA concentration data yields deceptively low rates, particularly at low concentrations of triGalUA, where hydrolysis of product diGalUA causes diGalUA concentrations to decrease compared to the concentration of diGalUA resulting from triGalUA 
hydrolysis alone. Thus, quantification by monoGalUA concentration alone results in an erroneously low $K_{\mathrm{m}}$ value $(3.2 \mu \mathrm{M})$ and quantification by diGalUA concentration alone results in an erroneously high $K_{\mathrm{m}}$ value $(26.7 \mu \mathrm{M})$. Applying Eq. (6), which uses both the monoGalUA and diGalUA concentration data, results in an intermediate $K_{\mathrm{m}}$ value $(15.8 \mu \mathrm{M})$.

The determined $K_{\mathrm{m}}$ value of TtGH 28 acting on triGalUA is small $(15.8 \mu \mathrm{M})$, and the detection of products is limited $(3 \mu \mathrm{M})$. This necessitates that a significant portion of substrate is consumed for quantification of products, with a greater percentage consumed at low concentrations of substrate compared to higher concentrations. For example, at the lowest concentration used in this study $(29 \mu \mathrm{M})$, the percentage of triGalUA converted to product was about $18 \%$, whereas the percent conversion of triGalUA decreases to less than 5\% when triGalUA concentrations are greater than $0.3 \mathrm{mM}$ (Fig. 2). Thus, at a low concentration of triGalUA, the concentration of diGalUA produced is significantly large compared to the triGalUA in the reaction. Under these conditions, when the $k_{\text {cat }} / K_{\mathrm{m}}$ values of the enzyme for the dimer and trimer are similar as in this study $\left(k_{\text {cat }} / K_{\mathrm{m}}\right.$ values $1010 \mathrm{~s}^{-1} \mathrm{mM}^{-1}$ and $603 \mathrm{~s}^{-1} \mathrm{mM}^{-1}$, respectively), the dimer will also be hydrolyzed and contribute significantly to the concentration of product monomer. It is under these conditions (high percent conversions of the starting substrate trimer and similar $k_{\mathrm{cat}} / K_{\mathrm{m}}$ values for the trimer and the product dimer) that the use of Eq. (6) becomes necessary to correct for the contribution of dimer hydrolysis when determining the hydrolysis of the trimer. Notably, the need for Eq. (6) diminishes if the $k_{\text {cat }} / K_{\mathrm{m}}$ of the enzyme for the dimer is much lower than the $k_{\mathrm{cat}} / K_{\mathrm{m}}$ for the trimer, as the dimer concentration resulting from trimer hydrolysis will not be large enough for significant hydrolysis of product dimer to occur. The problem can also be avoided if the assay method utilized is sufficiently sensitive to 
analyze samples with very low percentage conversions of the substrate trimer such that product dimer concentrations are very low.

In summary, the method derived here resulting in Eq. (6) resolves some of the difficulty of determining by discontinuous assay the catalytic rates of exo-hydrolases acting on starting substrate trimer as well as product dimer. This method is particularly necessary when the assay method requires high percent conversions of the starting substrate and the enzyme studied has similar $k_{\text {cat }} / K_{\mathrm{m}}$ values for the starting substrate and the product; the utility of the method is demonstrated for the polygalacturonase $\mathrm{TtGH} 28$. The method is expanded for starting substrates of any length with Eq. (9).

\section{Acknowledgments}

This work was supported by United States Department of Agriculture (USDA) CRIS 362041000-118-00D (D.B.J. and J.R.S.), and USDA CRIS 5325-41000-049-00 and National Institute of Food and Agriculture grant 2012-03998 (C.C.L. and K.W.). This research was also supported in part by an appointment to the Agricultural Research Service (ARS) Research Participation Program administered by the Oak Ridge Institute for Science and Education (ORISE) through an interagency agreement between the U.S. Department of Energy (DOE) and the USDA (J.R.S.). ORISE is managed by Oak Ridge Associated Universities (ORAU) under DOE contract number DE-AC05-06OR23100. All opinions expressed in this paper are the authors' and do not necessarily reflect the policies and views of USDA, ARS, DOE, or ORAU/ORISE. The mention of firm names or trade products does not imply that they are endorsed or recommended by the USDA over other firms or similar products not mentioned. USDA is an equal opportunity provider and employer. 


\section{Figure legends}

Fig. 1. Steady state kinetics of TtGH28 acting on triGalUA at $\mathrm{pH} 6.0$ and $40{ }^{\circ} \mathrm{C}$ with three different quantification methods for initial rates. Data were collected in duplicate and are fit to Eq. (1). The fitted values obtained for TtGH28 acting on triGalUA are $k_{\text {cat }} 9.62 \pm 0.19 \mathrm{~s}^{-1}, k_{\text {cat }} / K_{\mathrm{m}}$ $3020 \pm 1120 \mathrm{~s}^{-1} \mathrm{mM}^{-1}, K_{\mathrm{m}} 3.2 \pm 1.2 \mu \mathrm{M}, K_{\mathrm{si}} 15.3 \pm 2.1 \mathrm{mM}$ for quantification by monoGalUA concentration; $k_{\text {cat }} 9.60 \pm 0.19 \mathrm{~s}^{-1}, k_{\text {cat }} / K_{\mathrm{m}} 360 \pm 27 \mathrm{~s}^{-1} \mathrm{mM}^{-1}, K_{\mathrm{m}} 26.7 \pm 2.4 \mu \mathrm{M}$, and $K_{\mathrm{si}} 17.9 \pm 2.3$ $\mathrm{mM}$ for quantification by diGalUA concentration; and $k_{\text {cat }} 9.51 \pm 0.17 \mathrm{~s}^{-1}, k_{\text {cat }} / K_{\mathrm{m}} 603 \pm 54 \mathrm{~s}^{-}$ ${ }^{1} \mathrm{mM}^{-1}, K_{\mathrm{m}} 15.8 \pm 1.6 \mu \mathrm{M}$, and $K_{\mathrm{si}} 17.7 \pm 2.1 \mathrm{mM}$ for the combined quantification method using Eq. (6).

Fig. 2. For TtGH28 acting on triGalUA, the percent conversion of triGalUA and the percent of monomer product arising from hydrolysis of diGalUA are shown at the concentration of triGalUA in the reaction. 


\section{References}

[1] H. Kawaguchi, T. Hasunuma, C. Ogino, A. Kondo, Bioprocessing of bio-based chemicals produced from lignocellulosic feedstocks, Current Opinion in Biotechnology, 42 (2016) 30-39. [2] V. Lombard, H. Golaconda Ramulu, E. Drula, P.M. Coutinho, B. Henrissat, The carbohydrate-active enzymes database (CAZy) in 2013, Nucleic Acids Research, 42 (2014) D490-D495.

[3] K. Wagschal, D.B. Jordan, C.C. Lee, A. Younger, J.D. Braker, V.J. Chan, Biochemical characterization of uronate dehydrogenases from three Pseudomonads, Chromohalobacter salixigens, and Polaromonas naphthalenivorans, Enzyme and Microbial Technology, 69 (2015) $62-68$.

[4] F.A. Isherwood, Y.T. Chen, L.W. Mapson, Synthesis of L-ascorbic acid in plants and animals, The Biochemical Journal, 56 (1954) 1-15.

[5] H.C.M. Kester, M.A. Kusters-Van Someren, Y. Müller, J. Visser, Primary structure and characterization of an exopolygalacturonase from Aspergillus tubingensis, European Journal of Biochemistry, 240 (1996) 738-746.

[6] K. Li, K. Meng, X. Pan, R. Ma, P. Yang, H. Huang, B. Yao, X. Su, Two thermophilic fungal pectinases from Neosartorya fischeri P1: Gene cloning, expression, and biochemical characterization, Journal of Molecular Catalysis B: Enzymatic, 118 (2015) 70-78.

[7] A. Maller, T.M. da Silva, A.R.D.L. Damásio, I.Y. Hirata, J.A. Jorge, H.F. Terenzi, M.L.T.M. Polizeli, Functional properties of a manganese-activated exo-polygalacturonase produced by a thermotolerant fungus Aspergillus niveus, Folia Microbiologica, 58 (2013) 615-621. 
[8] J.A. Mertens, Kinetic properties of two Rhizopus Exo-polygalacturonase enzymes hydrolyzing galacturonic acid oligomers using isothermal titration calorimetry, Applied Biochemistry and Biotechnology, 170 (2013) 2009-2020.

[9] J.A. Mertens, M.J. Bowman, Expression and characterization of fifteen Rhizopus oryzae 99880 polygalacturonase enzymes in Pichia pastoris, Current Microbiology, 62 (2011) 1173-1178. [10] Y. Pauchet, R. Kirsch, S. Giraud, H. Vogel, D.G. Heckel, Identification and characterization of plant cell wall degrading enzymes from three glycoside hydrolase families in the cerambycid beetle Apriona japonica, Insect Biochemistry and Molecular Biology, 49 (2014) 1-13. [11] C. Tari, N. Dogan, N. Gogus, Biochemical and thermal characterization of crude exopolygalacturonase produced by Aspergillus sojae, Food Chemistry, 111 (2008) 824-829. [12] Y. Chen, D. Sun, Y. Zhou, L. Liu, W. Han, B. Zheng, Z. Wang, Z. Zhang, Cloning, expression and characterization of a novel thermophilic polygalacturonase from Caldicellulosiruptor bescii DSM 6725, International Journal of Molecular Sciences, 15 (2014) $5717-5729$.

[13] S. Kar, R.C. Ray, Purification, characterization and application of thermostable exopolygalacturonase from Streptomyces erumpens mtcc 7317, Journal of Food Biochemistry, 35 (2011) 133-147.

[14] L.D. Kluskens, G.J.W.M. Van Alebeek, J. Walther, A.G.J. Voragen, W.M. De Vos, J. Van Der Oost, Characterization and mode of action of an exopolygalacturonase from the hyperthermophilic bacterium Thermotoga maritima, FEBS Journal, 272 (2005) 5464-5473. [15] C.H. Liao, L. Revear, A. Hotchkiss, B. Savary, Genetic and biochemical characterization of an exopolygalacturonase and a pectate lyase from Yersinia enterocolitica, Canadian Journal of Microbiology, 45 (1999) 396-403. 
[16] J. Parisot, V. Langlois, V. Sakanyan, C. Rabiller, Cloning expression and characterization of a thermostable exopolygalacturonase from Thermotoga maritima, Carbohydrate Research, 338 (2003) 1333-1337.

[17] D.B. Pedrolli, E.C. Carmona, Purification and characterization of the exopolygalacturonase produced by Aspergillus giganteus in submerged cultures, Journal of Industrial Microbiology and Biotechnology, 37 (2010) 567-573.

[18] K. Sawada, A. Suzumatsu, T. Kobayashi, S. Ito, Molecular cloning and sequencing of the gene encoding an exopolygalacturonase of a Bacillus isolate and properties of its recombinant enzyme, Biochimica et Biophysica Acta - General Subjects, 1568 (2001) 162-170.

[19] K. Wagschal, J. Rose Stoller, V.J. Chan, C.C. Lee, A.A. Grigorescu, D.B. Jordan, Expression and characterization of hyperthermostable exo-polygalacturonase $\mathrm{TtGH} 28$ from Thermotoga thermophilus, Molecular Biotechnology, 58 (2016) 509-519.

[20] R.J. Leatherbarrow, GraFit 5, Erithacus Software, Ltd., Horley, U.K. (2004). 


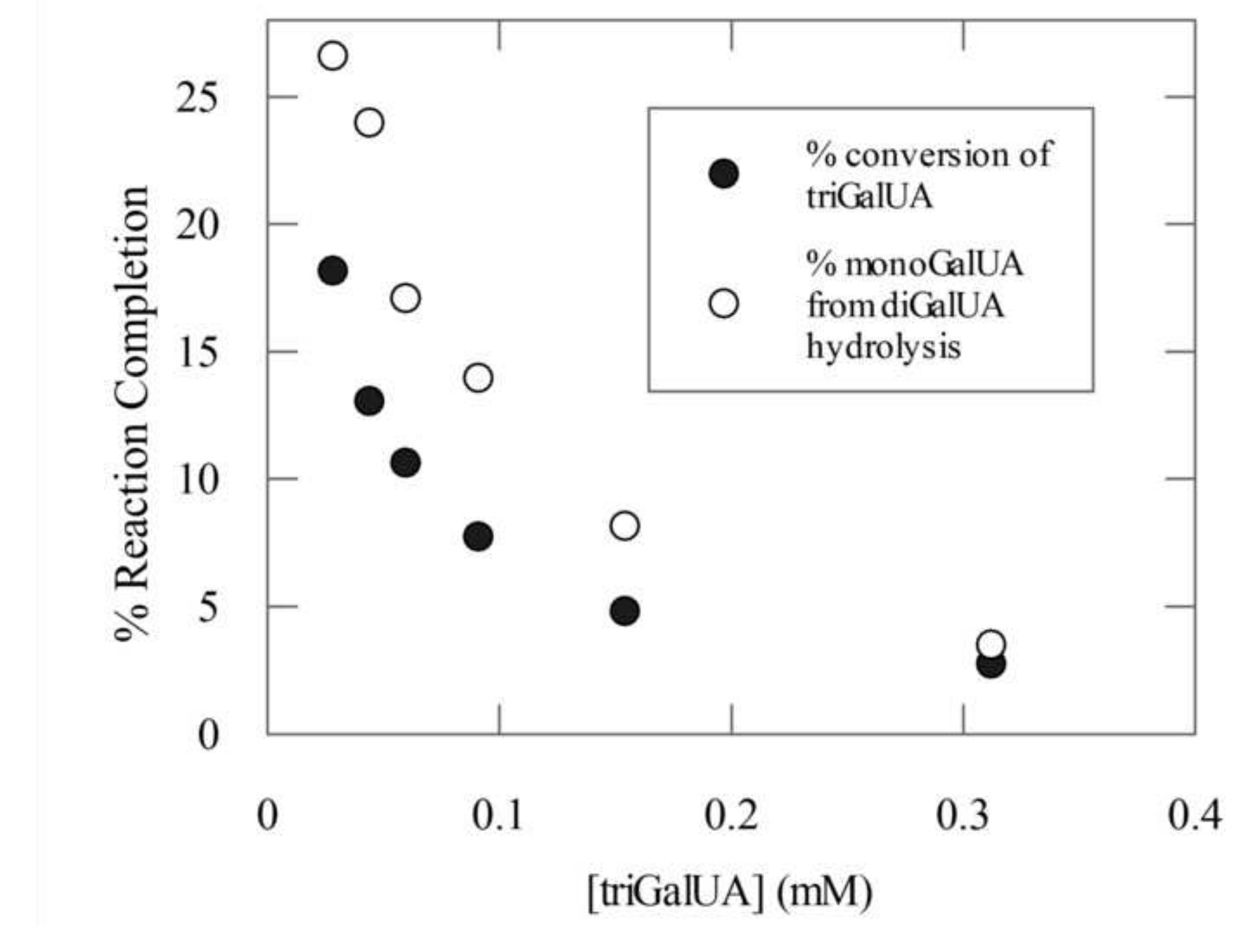

